\title{
Economic evaluation of personalized medicine: a call for real-world data
}

\author{
Robert Terkola $^{1,2} \cdot$ Fernando Antoñanzas ${ }^{3} \cdot$ Maarten Postma $^{2,4,5}$
}

Published online: 5 April 2017

(c) Springer-Verlag Berlin Heidelberg 2017

\section{Introduction}

In the last decade, we have witnessed a change in healthcare management, with 'personalized medicine' as the new paradigm. Also referred to as 'individualized', 'stratified', or 'precision' medicine, this new paradigm for the diagnosis and management of disease aims to tailor protocols and treatments to the personal and medical characteristics of patients. Personalized medicine (PM) has rapidly penetrated European Union (EU) healthcare systems. Although there is no universally accepted definition, the Horizon 2020 Advisory Group of the EU has defined PM as "a medical model using characterization of individuals' phenotypes and genotypes (e.g., molecular profiling, medical imaging, lifestyle data) for tailoring the right therapeutic strategy for the right person at the right time, and/or to determine the predisposition to disease and/or to deliver timely and targeted prevention" [1].

Fernando Antoñanzas

fernando.antonanzas@unirioja.es

University of Florida-College of Pharmacy, Gainesville, USA

2 Unit of PharmacoTherapy, -Epidemiology and -Economics (PTEE), Department of Pharmacy, University of Groningen, Groningen, The Netherlands

3 Department of Economics, University of La Rioja, Logrono, Spain

4 Institute of Science in Healthy Aging and Healthcare (SHARE), University Medical Center Groningen (UMCG), University of Groningen, Groningen, The Netherlands

5 Department of Epidemiology, University Medical Center Groningen (UMCG), University of Groningen, Groningen, The Netherlands
So far, this concept been translated to practice predominantly through the use of genetic diagnostic tests connected to a distinct molecular characteristic. Notably, these diagnostics are usually associated with a class of drugs and are typically not strictly restricted to a specific drug or to specific uses within an approved label or indication. Currently, in the USA alone, 26,000 genetic diagnostic tests are available, covering over 3600 genes [2], making PM one of the areas in global health care with the fastest growth potential. This new PM paradigm has triggered many publications across multiple scientific areas, totaling about 2500 in the year 2012 [3] and double that number in 2016 [4]. PM has the potential to allow patients to receive drugs specific to their individual disease, and to increase the efficiency of the healthcare system.

\section{Economic evaluation in personalized medicine}

In many countries, economic evaluation is used as a practical tool for making decisions about the introduction and implementation of health technologies. Economic evaluation requires data on health outcomes and resource utilization associated with a technology to be combined in an analytical model to calculate the typical cost per life year or quality-adjusted life year (QALY) gained. PM is the next challenge for economic evaluation, and there is a growing number of published economic evaluations and reviews in this area.

This experience of performing economic evaluations of medical technologies in this area has generated a set of knowledge about how to develop new models incorporating the complex diagnostic criteria needed in PM, and about how to measure health outcomes and well-being measures related to the new information derived from 
genetic tests. There remain several unsolved questions, previously described by a number of authors, that pose challenges to the application of economic evaluation to PM [5-8]. In particular, the main elements that require further research and consensus include [5-8]:

- The dependence of the results of economic evaluations in PM on the inclusion of healthcare costs and health outcomes derived from testing inaccuracies (i.e., falsenegative, false-positive), which are subject to population variability and cannot easily be extrapolated from the results of clinical trials.

- The lack of generalized and integrated observational 'real-world' databases for costs and health outcomes associated with PM technologies.

- The necessity for fine-tuning data on costs of tests, with typically a number of tests, at varying costs, able to identify a particular genetic characteristic.

- The failure of QALYs to fully capture the subtleties of PM health outcomes and related well-being.

- The complexity needed in analytical decision models to incorporate the additional testing steps in the PM treatment algorithm (e.g., single vs. sequential tests), alongside all the other variables and parameters involved in any economic evaluation, resulting in a higher level of uncertainty around the final incremental cost-effectiveness ratio (ICER).

As a summary, we believe, together with other authors $[7,9,10]$, that the lack of high-quality data on costs and health outcomes is the major reason why there is no clear evidence for the value of PM in terms of cost-effectiveness. We also note that even if more data were available, measuring the value of PM is inherently challenging, as there is still no commonly accepted definition of value [11].

Cost-effectiveness is based on the effectiveness achieved by the assessed technology. Usually, at the time of performing an initial economic evaluation, the available information on health outcomes comprises efficacy data from randomized controlled trials (RCTs) rather than realworld effectiveness. At this stage, the efficacy data used in evaluations of PM technologies are similar to those used in the assessment of other medicines. However, in the case of traditional medicines, once a product is in clinical use the data generated in clinical trials can be compared with the effectiveness results seen in real-world practice, validating (or challenging) the economic evaluation results already achieved. Notably, when real-world data in oncology have been collected and systematically compared with clinical trial evidence the results in practice have sometimes been aligned with the clinical trial setting, but on other occasions outcomes have deviated from expectations. Similarly, with the introduction of a new approach to medicine- - such as $\mathrm{PM}-\mathrm{it}$ is desirable to have at our disposal real-world studies showing the effectiveness of the new technologies. Compared with traditional medicine, implementation of PM requires additional tests and more complex protocols to achieve the anticipated health gains demonstrated in clinical trials. Additionally, clinical trial evidence for PM therapies may be less robust than for traditional medicines, as the evidence base may be only a subgroup of a larger trial, increasing the importance of gathering complementary data in clinical practice. If PM is to replace the traditional approach to medicine, successful incorporation of this additional complexity into real-world practice must be confirmed. As well as validating the results of economic evaluations, high-quality, real-world PM data would clarify the efficiency of new technologies. However, despite the need to improve the quality of economic evaluations in $\mathrm{PM}$, to the best of our knowledge, the use of real-world data in this area is still limited.

\section{Lack of real-world evidence of personalized medicine effectiveness}

The correspondence between efficacy data from pre-authorization clinical trials, upon which economic evaluations are usually based, and real-world effectiveness has been analyzed by the authors of this editorial, as part of an investigation into whether the benefits of PM therapies reported in RCTs are maintained when therapies are used in routine practice. In other words, we aimed to understand whether the economic evaluation results of PM technologies were accurate and applicable to real-world medical practice.

To explore the status quo in more detail, we have conducted a systematic search of the literature, which identified an absence of studies comparing clinical trial results with real-world outcomes in the PM field. For many experts, this is an unexpected finding. The search strategy was limited to studies published in the last 6 years that specifically compared outcomes in real-world practice with those in RCTs, as we considered this to be sufficient to determine the current situation. MEDLINE, Embase, and Embase Alert databases were searched on July 20, 2016 using multiple free-text terms covering personalized medicine, stratified medicine and precision medicine, combined with terms referring to real-world evidence. In summary, 2250 articles were retrieved in the database searches, of which ten were identified as being potentially relevant. However, at the full-text review stage, no study was identified that presented relevant data comparing PM clinical trial results with real-world outcomes, confirming our hypothesis that evidence in this area is lacking.

Although there is much interest in the potential of PM, particularly in oncology, it is apparent that there is a 
paucity of literature addressing the question of how the benefits of PM therapies reported in RCTs translate into outcomes in real-world clinical practice. Further investigation of this question may require an analysis of observational study findings for individual therapies, and comparison with the results of the pivotal RCTs.

\section{Conclusions}

Together with other authors [2], we acknowledge that measurement of the value offered by PM is crucial. To do so effectively will require a paramount effort to comprehend the different perspectives of the concept of value in health. The cornerstone of understanding the value of new medical strategies such as PM is assessment of the precision achieved in the real world, i.e., the extent to which PM enables each patient to receive the right drug at the right time. Challenges to the precision of PM in this respect are that the diagnostic tests needed for PM drugs are typically prone to false-negative and false-positive results, and that further uncertainty often exists about the incidence of diseases (defined at the molecular level). These aspects of PM can combine with the difficulties in incorporating pricing, cost of illness, and burden of disease into economic evaluation to negatively impact pharmacoeconomic outcomes.

One partial solution to the resultant uncertainty is scenario analysis, which can be used to generate an interval of possible values for the ICER. However, as the use of PM increases, health economists should be ambitious and aim to populate our models with the most accurate data possible, so that the evaluation results can be used confidently during the decision-making processes. Therefore, health economists should strongly support the generation of precise and accurate data. Such a call to action should be directed to responsible stakeholders who are in the position to foster the implementation of real-world big data generation systems to support their installation as an integrated part of our healthcare system. Coordination between regulators including the European Medicines Agency and the US Food and Drug Administration is also important to ensure that diagnostic tests meet consistent standards for quality and accuracy, while more research is needed to determine how differences between testing methodologies (for example, fluorescent in situ hybridization and nextgeneration sequencing) affect the efficiency of PM. Overall, we consider that health outcomes data are a major determinant of the quality of the results of economic evaluation studies. In addition to overcoming other limitations and challenges in the economic evaluation of PM therapies, as described above, establishment of methods for the generation of high-quality, real-world outcome data, using validated definitions of all explored variables, is an essential step towards understanding the potential of this promising new approach to medicine.

Acknowledgements Editorial assistance in the preparation of this article was provided by Paul Overton (independent medical writer) in accordance with Good Publication Practice (GPP3) guidelines (http:// www.ismpp.org/gpp3). Research and editorial support were funded by Novartis Pharma, Basel under the AGORA initiative (Advisory Group On Reimbursement and Access, a European Think Tank which aims to optimize access for patients to innovative treatments). All opinions described in this article are solely those of the authors. Robert Terkola, Fernando Antoñanzas, and Maarten Postma have received honoraria and travel support related to this study from Novartis Pharma AG under the AGORA initiative.

\section{References}

1. European Union: Council conclusions on personalised medicine for patients. http://eur-lex.europa.eu/legal-content/EN/TXT/PDF/ ?uri=CELEX:52015XG1217(01)\&from=EN (2015). Accessed 6 March 2017

2. Akhmetov, I., Bubnov, R.V.: Assessing value of innovative molecular diagnostic tests in the concept of predictive, preventive, and personalized medicine. EPMA J. 6, 19 (2015)

3. O'Donnell, J.C.: Personalized medicine and the role of health economics and outcomes research: issues, applications, emerging trends, and future research. Value Health 16(6 Suppl), S1-S3 (2013)

4. National center for biotechnology information: PubMed. https:// www.ncbi.nlm.nih.gov/pubmed. Accessed 6 March 2017

5. Annemans, L., Redekop, K., Payne, K.: Current methodological issues in the economic assessment of personalized medicine. Value Health 16(6 Suppl), S20-S26 (2013)

6. Faulkner, E., Annemans, L., Garrison, L., Helfand, M., Holtorf, A.P., Hornberger, J., Hughes, D., Li, T., Malone, D., Payne, K., Siebert, U., Towse, A., Veenstra, D., Watkins, J., Personalized Medicine, D., Reimbursement Working, G.: Challenges in the development and reimbursement of personalized medicine-payer and manufacturer perspectives and implications for health economics and outcomes research: a report of the ISPOR personalized medicine special interest group. Value Health 15(8), 1162-1171 (2012)

7. Hatz, M.H., Schremser, K., Rogowski, W.H.: Is individualized medicine more cost-effective? A systematic review. Pharmacoeconomics 32(5), 443-455 (2014)

8. Postma, M.J., Boersma, C., Vandijck, D., Vegter, S., Le, H.H., Annemans, L.: Health technology assessments in personalized medicine: illustrations for cost-effectiveness analysis. Exp. Rev. Pharmacoecon. Outcomes. Res. 11(4), 367-369 (2011)

9. Deverka, P.A.: Pharmacogenomics, evidence, and the role of payers. Public. Health. Genom. 12(3), 149-157 (2009)

10. Deverka, P.A., Vernon, J., McLeod, H.L.: Economic opportunities and challenges for pharmacogenomics. Annu. Rev. Pharmacol. Toxicol. 50, 423-437 (2010)

11. Antonanzas, F., Terkola, R., Postma, M.: The value of medicines: a crucial but vague concept. Pharmacoeconomics 34(12), 1227-1239 (2016) 